\title{
AN UNUSUAL PRESENTATION OF MESENTERIC PANNICULITIS
}

\author{
doi: $10.1590 / \mathrm{S1807-59322008000600023}$
}

Teresa Cristina A. Ferrari, ${ }^{\mathrm{I}}$ Carolina M. Couto, ${ }^{\mathrm{I}}$ Tatiane S. Vilaça, ${ }^{\mathrm{I}}$ Marcelo A. P. Xavier, ${ }^{\mathrm{II}}$ Luciana C. Faria ${ }^{\mathrm{I}}$

\section{INTRODUCTION}

Mesenteric panniculitis is a rare disorder characterized by a tumor-like expansion of the mesentery due to variable degrees of fat necrosis, chronic inflammation and fibrosis. Its pathophysiology remains unclear despite the description of associations with inflammatory disorders or malignancies, especially lymphomas. ${ }^{1}$ The disease remains asymptomatic in $30-50 \%$ of cases, and the most common clinical presentations are abdominal pain, palpable abdominal mass or intestinal obstruction. ${ }^{1,2}$ Most patients present a benign, slowly progressive course, and the outcome of the disease is usually favorable. ${ }^{1,2}$ Nevertheless, in about $20 \%$ of patients, mesenteric panniculitis is associated with significant morbidity and a chronic debilitating course. ${ }^{3}$

We report a case of this poorly known entity, which presented as a systemic disease with severe constitutional manifestations and rapid deterioration of the general condition of the patient, which made diagnosis difficult.

\section{CASE DESCRIPTION}

A 36-year-old previously healthy man was admitted to our institution with daily fever (up to $39.3^{\circ} \mathrm{C}$ ), chills, significant weight loss (more than $10 \%$ of his normal weight) and severe impairment of his general condition lasting for six weeks before admission. He denied abdominal pain and gastrointestinal symptoms. Physical examination was unremarkable except for a hard and slightly painful mass in the left upper abdomen. Laboratory investigations showed hemoglobin levels at $10.2 \mathrm{~g} / \mathrm{dL}$, a white blood cell count of $27,300 / \mathrm{mm}^{3}$ with a shift to the left, a platelet cell count of $640,000 / \mathrm{mm}^{3}$, an erythrocyte sedimentation rate (ESR) of $100 \mathrm{~mm} / \mathrm{h}, \mathrm{h}$-reactive protein levels at $192 \mathrm{mg} / \mathrm{L}$ and serum albumin levels of $2.2 \mathrm{~g} / \mathrm{dL}$. Bilirubin levels, hepatic and pancreatic enzyme levels and renal function tests were normal. Blood cultures, the anti-HIV test and the tuberculin

\footnotetext{
I Department of Internal Medicine, Federal University of Minas Gerais, School of Medicine - Belo Horizonte/MG, Brazil.

"Department of Pathology and Legal Medicine, Federal University of Minas Gerais, School of Medicine - Belo Horizonte/MG, Brazil.

Email: tferrari@medicina.ufmg.br

Tel.: 55313409.9746
}

skin test (PPD) were negative, and the chest $\mathrm{x}$-ray showed no abnormalities. Abdominal CT scan revealed a softtissue mass in the left upper abdomen, as well as increased attenuation of mesenteric and perinephric fat on the left.

During hospitalization, the patient continued to have daily fever and significant weight loss. Repeated physical examination revealed marked variation of the sensitivity, location and size of the abdominal mass. Laparoscopy identified a solid tumor measuring approximately $10 \mathrm{~cm}$ in diameter located in the mesentery, and a biopsy of this lesion showed nonspecific inflammatory changes. Since a diagnosis was not achieved, the patient was submitted to exploratory laparotomy, which disclosed a hard mass involving the transverse mesocolon at the level of the splenic flexure adhered to the bowel loops. Light grey necrotic tissue was observed upon the sectioning of the adhesions. A biopsy of the mass was performed. Histopathological examination of this material showed wellvascularized fibroadipose tissue with areas of adiponecrosis and nests of foamy macrophages, areas of recent hemorrhage, foci of mononuclear cell inflammatory infiltrate and proliferation of bundle-forming fibroblasts (Figure 1). All tests and cultures for fungi and mycobacteria were negative.

Due to the progressive worsening of the patient, we started prednisone administration $(60 \mathrm{mg} /$ day $)$. The fever disappeared within a few days, and the patient experienced a steady progressive improvement. A regression of the abdominal mass was also observed. After two weeks, the corticosteroid was gradually tapered until its complete discontinuation in five months. Blood cell counts, ESR, $\mathrm{C}$-reactive protein and albumin returned to the normal values within several weeks. An abdominal CT performed six months after the beginning of the treatment showed a complete disappearance of the abnormalities. Two years later, the patient remains in good health with no recurrence.

\section{DISCUSSION}

Mesenteric panniculitis is a rare disorder of unknown etiology that is likely underdiagnosed. Some associated conditions have already been reported: malignancies (lymphoma and carcinoma), autoimmunity, infection, trauma and a history of previous surgery. However, definitive causal relationships have never been confirmed. ${ }^{1,3}$ 


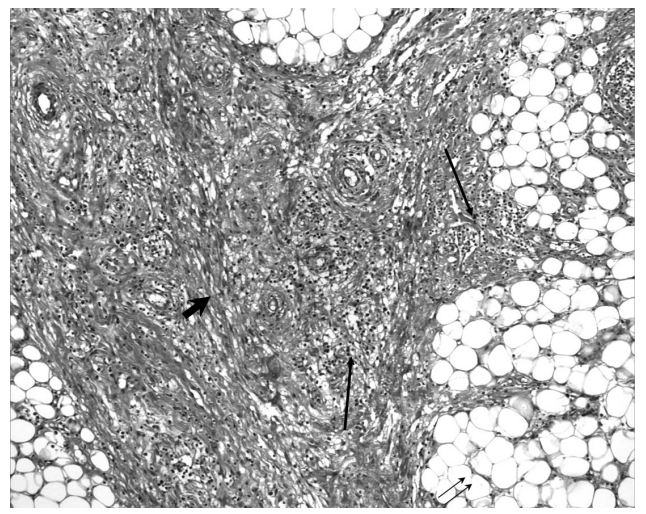

Figure 1 - Fibroadipose tissue with areas of adiponecrosis (double arrows), foci of mononuclear cell inflammatory infiltrate (long arrows) and proliferation of bundle-forming fibroblasts (large arrow) (Hematoxylin-eosin, $\times 100)$

The condition predominantly occurs in males and is more frequent between the $6^{\text {th }}$ and $7^{\text {th }}$ decades of life. ${ }^{3} \mathrm{~A}$ wide variety of gastrointestinal and systemic manifestations have been associated with this disorder; however, the most common presentations are abdominal pain, bloating and distension, diarrhea, abdominal mass and intestinal obstruction. ${ }^{1-5}$ Other reported symptoms include weight loss, constipation, anorexia and fever. ${ }^{2,3}$ Presentations such as fever of unknown origin and protein-losing enteropathy have also been described. Laboratory parameters are frequently normal; however, an increased erythrocyte sedimentation rate has been noted. ${ }^{3}$

The present case showed some peculiarities that made its diagnosis difficult. Our patient was younger than most of the reported cases and his clinical picture, dominated by severe systemic symptoms and rapidly progressive clinical deterioration, along with the presence of significant leukocytosis and elevated levels of the markers of acute phase response, suggested a systemic disease and made the diagnosis difficult. Another interesting characteristic of the present case was the marked variation in the characteristics of the abdominal mass upon physical examination performed on different occasions.

Abdominal CT is currently being proposed as an effective method for diagnostic evaluation. Two CT findings are considered somewhat specific for this disorder: a "fat ring sign" that reflects the preservation of fat around the mesenteric vessels, and the presence of a "tumoral pseudocapsule," which is detected in $50 \%$ of patients. ${ }^{6}$ In a recently published series of 92 patients, the main CT findings were a single soft- tissue mass in the root of the mesentery that often contained calcification (61\% of cases) and a subtly increased density of the mesenteric fat, with or without discrete soft-tissue masses, suggesting mild mesenteric fibrosis or inflammation (34\% of cases). ${ }^{3}$ In the present case, the CT findings contributed little to the diagnosis due to their lack of specificity.

The definite diagnosis of mesenteric panniculitis is established by biopsy. Variable combinations of foci of fat degeneration and necrosis, a nonspecific and predominantly lymphocytic inflammatory infiltrate and fibrosis have been reported. ${ }^{2,3}$ Based on the predominant histological findings, the disease has been called mesenteric lipodystrophy, mesenteric panniculitis or sclerosing mesenteritis, according to the predominance of adiponecrosis, inflammation or fibrosis, respectively. Some evidence suggests that these three morphological lesions represent a single clinical entity. ${ }^{2}$ In the present case, inflammation was the most prominent feature, although adiponecrosis and areas of fibrosis were observed (Figure 1).

The differential diagnosis of mesenteric panniculitis is broad and includes all disorders that can affect the mesentery. The most common are lymphoma, well-differentiated liposarcoma, peritoneal carcinomatosis, carcinoid tumor, retroperitoneal fibrosis, lipoma, mesenteric desmoid tumor, mesenteric inflammatory pseudotumor, mesenteric fibromatosis and mesenteric edema. ${ }^{1,7}$

The course of mesenteric panniculitis is favorable in most cases, ${ }^{1,2}$ because the disease usually progresses slowly and subsides spontaneously. Nevertheless, in about $20 \%$ of patients, it is associated with significant morbidity and a chronic debilitating course. ${ }^{3}$

In our patient, the severity and rapid progression of the symptoms led us to prescribe prednisone, which had an excellent response. Other authors have also reported a beneficial effect of corticosteroid therapy. ${ }^{3,5}$ Other empirically proposed therapy options include tamoxifen, azathioprine, cyclophosphamide, oral progesterone and thalidomide. Surgical resection is generally not recommended and has no beneficial effects, except in cases of intestinal obstruction and other complications, such as ischemia.

In conclusion, mesenteric panniculitis should be considered in the differential diagnosis of patients with severe systemic manifestations and elevated markers of the acute phase response of unknown etiology, especially in the presence of abdominal symptoms. In these cases, the response to steroid therapy may be excellent.

\section{REFERENCES}

1. Piessen G, Mariette C, Triboulet JP. Mesenteric panniculitis. Annal Chir 2006;131:85-90.

2. Emory TS, Monihan JM, Carr N J, Sobin, LH. Sclerosing Mesenteritis, Mesenteric Panniculitis and Mesenteric Lipodystrophy: A Single Entity? Am J Surg Pathol. 1997;21:392-98.

3. Akram S, Pardi DS, Schaffner JA, Smyrk TC. Sclerosing mesenteritis: clinical features, treatment, and outcome in ninety-two patients. Clin Gastroenterol Hepatol. 2007;5:589-96.

4. Béchade D, Durand X, Desramé J, Rambelo A, Corberand D, Baranger $\mathrm{B}$, et al. Etiologic spectrum of mesenteric panniculitis: report of 7 cases.
Rev Med Intern. 2007;28:289-95.

5. Tytgat GN, Roozendaal K, Winter W, Esseveld MR. Successful treatment of a patient with retractile mesenteritis with prednisone and azathioprine. Gastroenterology. 1980;79:352-56.

6. Horton KM, Lawler LP, Fishman EK. CT findings in sclerosing mesenteritis (panniculitis): spectrum of disease. Radiographics. 2003:23:1561-67.

7. Kelly JK, Hwang WS. Idiopathic retractile (sclerosing) mesenteritis and its differential diagnosis. Am J Surg Pathol. 1989;13:513-21. 